\section{Tracking JAKs in spondyloarthritis: rationale and expectations}

\author{
Corinne Miceli-Richard, ${ }^{1,2}$ Maxime Dougados 1,2,3
}

In this issue, Van der Heijde and colleagues ${ }^{1}$ report the results of a randomised, prospective, placebo-controlled, dose-ranging trial evaluating the short-term (12 weeks) symptomatic effect of a Janus kinase (JAK) inhibitor, tofacitinib. This well-conducted and optimally presented trial raised two important points: (1) a potential role of the JAK/signal transducer and activator of transcription (STAT) pathway in the pathophysiology of axial spondyloarthritis ( $\mathrm{SpA}$ ) and (2) the expected results allowing to anticipate that such compounds might be included in the armamentarium for axial SpA.

JAKs are important intracellular tyrosine kinases involved in the signalling of many cytokines, chemokines and growth factors. JAKs play a critical role in both innate and adaptive immunity, which has justified the large number of JAK inhibitors developed in the past decades in the field of cancer but also rheumatology. ${ }^{12}$ JAKs bind to the intracellular domain of a broad range of receptors. Cytokines binding to their receptor leads to JAK phosphorylation, which allows for recruitment and phosphorylation of STAT protein via their Src Homology 2 domains. STATs then form heterodimers or homodimers that translocate to the nucleus and bind to specific DNA domains, ultimately leading to gene transcription.

JAK inhibitors are small molecules that are orally given, an alternative route of administration to subcutaneous-delivered or intravenous-delivered biologics. JAK inhibition is expected to efficiently dampen the inflammation and activation of the immune system observed in several chronic inflammatory conditions such as rheumatoid arthritis, axial $\mathrm{SpA}$, psoriatic arthritis and psoriasis. In contrast to therapeutic antibodies engineered to neutralise one specific cytokine, such as

\footnotetext{
${ }^{1}$ Department of Rheumatology-Hôpital Cochin, Assistance Publique-Hôpitaux de Paris, Paris Descartes University, Paris, France; ${ }^{2}$ Immunoregulation Unit, Unité Mixte AP-HP/Institut Pasteur, Institut Pasteur, Paris, France; ${ }^{3}$ INSERM (U1153): Clinical Epidemiology and Biostatistics, PRES Sorbonne ParisCité, Paris, France

Correspondence to Professor Maxime Dougados, Hopital Cochin, Rheumatology, Université Paris Descartes, INSERM (U1153), Paris 75014, France; maxime.dougados@aphp.fr
}

tumour necrosis factor $\alpha(\mathrm{TNF} \alpha)$, or one specific cytokine receptor, such as interleukin 6 (IL-6) receptor, JAK inhibitors can have a broad range of cytokine inhibition. They have a downstream effect on cytokine receptor signalling in that different receptors signal through the recruitment of shared JAKs (eg, JAK1/tyrosine kinase 2 (TYK2) for the receptors of type 1 interferon (IFN) and the IL-10 family of cytokines or JAK1/JAK2/TYK2 for the receptors of IL-6, IL-27 and IL-35). ${ }^{3}$

Nevertheless, this broad anticytokine effect expected from JAK/STAT inhibition faces a differential range of efficacy of JAK inhibitors depending on the inflammatory condition for which they have been evaluated in phase II/III trials. As an example, tofacitinib has been approved for rheumatoid arthritis ${ }^{4-6}$ and has shown interesting profiles of efficacy with psoriasis $^{7}$ and psoriatic arthritis; ${ }^{8}$ in axial SpA, the results reported in this issue by Van der Heijde $e t a l^{1}$ are promising but can be seen as less impressive than in other rheumatological conditions. The specificity of JAK inhibition and the prominent cytokine pathways involved in each condition might explain these unequal response profiles. Recent data demonstrate that triggering the $\mathrm{T}$ helper 17 (Th17) cell pathway appears to be a good therapeutic option in axial SpA, psoriatic arthritis and psoriasis. Th17 differentiation and proliferation mainly rely on IL-23, a cytokine that signals through the IL-23 receptor, involving JAK2 and TYK2 as signal transducers. JAK2 activity is essential for haematopoiesis, and TYK2 is involved in signal transduction of IFN $\alpha / \beta$, IFN $\gamma$ and IL-12.

Thus, the development of a JAK inhibitor more specifically directed towards JAK2 and TYK2 to efficiently inhibit IL-23 might be theoretically associated with side effects such as impaired resistance to viral and bacterial pathogens and haematopoietic disturbance. Nevertheless, this kind of compound is not available until now, and tofacitinib is the only JAK inhibitor that has received regulatory approval in rheumatology. Indeed, tofacitinib is probably not the best JAK inhibitor to efficiently target the IL-23 pathway according to in vitro experiments demonstrating that tofacitinib inhibits JAK1 and
JAK3, with marginal inhibition of JAK2 and TYK2. ${ }^{3}$ Similar experiments have shown baricitinib with more specificity for JAK1 and JAK2 over JAK $3^{3}$ and should be, in this regard, more effective in inhibiting IL-23 signalling and the Th17 pathway, hypotheses that require demonstration in clinical trials.

In fact, outlining the different profiles of efficacy of JAK inhibitors to their more prominent action on a specific JAK is probably a reductive point of view. Cell signalling mediated by JAKs and STATS is highly complex, and response to a cytokine can differ depending on the cell owing to the various STATs and kinases involved. Therefore, the effect of JAK inhibitors should be ideally assessed in the target tissue involved in the treated condition: synovial membrane in rheumatoid arthritis or psoriatic arthritis ${ }^{9} 10$ and keratinocytes in psoriasis. ${ }^{11}$

Hence, predicting the efficacy of any JAK inhibitor in a given condition seems extremely difficult because of its own biased selectivity, the joint action of different JAKs on cell signalling and the cellspecific STATs engaged in the signalling cascade. Moreover, because of the various permissible heterodimeric pairings of Jak enzymes, the effects of inhibition on the pair by a Jak inhibitor selective for one member of the component enzymes in the heterodimer will be difficult to predict. JAK inhibition in SpA has promise, but the clinical evaluation of each of these compounds in the different clinical phenotypes of the disease is mandatory to better delineate their own scope of efficacy.

The interpretation of the clinical relevance of the findings from Van der Heijde and colleagues ${ }^{1}$ might differ according to different perspectives, particularly pharmaceutical companies in charge of developing a specific compound for a specific disease and clinicians managing disease in daily practice. In this article, specifically table 2, describing the 'Emax model-predicted ASAS20 response', the data observed did not reach the a priori decided target in terms of ASAS20 treatment response at week 12 . The observed ('actual' in the manuscript) results were in fact in line with or above expectations with $2 \mathrm{mg}$ and $5 \mathrm{mg}$ twice daily tofacitinib (eg, treatment effect greater than placebo of $10.7 \%$ and $39.6 \%$ ) but not the $10 \mathrm{mg}$ dose (eg, treatment effect greater than placebo of only $14.2 \%$ ). Such results might prompt the pharmaceutical company to make a 'no-go' decision on continuing development of this drug for this indication. The interpretation by clinicians might differ. 
Clinicians might look at the results for the collected outcome measures without considering any statistical $p$ value and consider the currently limited therapeutic options. Most clinicians consider the ASAS40 responder criteria more relevant than the ASAS20 criteria. In this respect, the results with tofacitinib $2 \mathrm{mg}$ and $5 \mathrm{mg}$ compared with placebo are at least as positive as the 'conventional' other biotherapies such as TNF inhibitors ${ }^{12-14}$ or IL-17 inhibitors. ${ }^{15}$ Moreover, the results for all collected outcome measures (eg, Ankylosing Spondylitis Disease Activity Score (ASDAS) responder and status criteria and Bath Ankylosing Spondylitis Disease Activity Index (BASDAI) responder criteria) corroborate the results observed for the Assessment of Spondylo Arthritis International Society (ASAS) response criteria. However, we must recognise that for all the symptomatic outcome variables, this trial failed to observe a clear dose-ranging effect with, in particular, a lower magnitude of treatment effect for the $10 \mathrm{mg}$ versus $5 \mathrm{mg}$ and $2 \mathrm{mg}$ doses. Such intriguing results might question the validity of the observed data.

In interpreting the findings of this trial, we could also consider the results observed for the outcome parameters evaluating the objective signs of inflammation such as MRI changes in inflammatory parameters observed both at the spine and sacroiliac joint level. For these variables, the data showed a clear difference versus placebo for the $5 \mathrm{mg}$ and $10 \mathrm{mg}$ doses and also a trend in favour of a dose-ranging effect.

One could consider that, if at the group level, the results are positive but not so impressive, some patients might benefit from this drug. This suggestion emphasises the importance of continuing our efforts to detect predisposing factors of a specific treatment response (eg, TNF inhibitors vs IL-17 inhibitors vs JAK inhibitors). The recognition of such predisposing factors will highly facilitate decision making by the rheumatologist in daily practice but also a better definition of the patients to be enrolled in clinical trials.

Finally, other important factors before considering a new treatment to be included in our armamentarium include safety profile, route of administration and cost. Therefore, here, we echo the opinion of Van der Heijde and colleagues in the last section of the manuscript: "JAK inhibitors may present a new mode of action for managing spondyloarthritis and could add to the currently limited options".

Contributors The two authors contributed equally and both of them meet the criteria for authorship.

Competing interests I have participated and I am participating at advisory boards organised by pharmaceutical companies such as Pfizer and Lilly to discuss the role of JAK inhibitors in axial SpA.

Provenance and peer review Commissioned; externally peer reviewed.

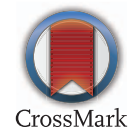

To cite Miceli-Richard C, Dougados M. Ann Rheum Dis 2017:76:1325-1326.

Received 19 January 2017

Accepted 21 February 2017

Published Online First 27 January 2017

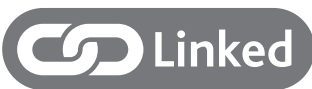

http://dx.doi.org/10.1136/annrheumdis-2016210322

Ann Rheum Dis 2017;76:1325-1326.

doi:10.1136/annrheumdis-2016-210886

\section{REFERENCES}

1 Van der Heijde D, Deodhar A, Wei JC, et al. Tofacitinib in patients with ankylosing spondylitis: a phase 2, 16-week, randomised, placebo-controlled, dose-ranging study. Ann Rheum Dis 2017;76:1340-7.

2 Kettle JG, Åstrand A, Catley M, et al. Inhibitors of JAK-family kinases: an update on the patent literature 2013-2015, part 1. Expert Opin Ther Pat 2017:27:127-43

3 Clark JD, Flanagan ME, Telliez JB. Discovery and development of Janus kinase (JAK) inhibitors for inflammatory diseases. J Med Chem 2014;57:5023-38.
4 Burmester GR, Blanco R, Charles-Schoeman C, et al. ORAL Step investigators. Tofacitinib (CP-690,550) in combination with methotrexate in patients with active rheumatoid arthritis with an inadequate response to tumour necrosis factor inhibitors: a randomised phase 3 trial. Lancet 2013;381:451-60.

5 van Vollenhoven RF, Fleischmann R, Cohen S, et al., ORAL Standard Investigators. Tofacitinib or adalimumab versus placebo in rheumatoid arthritis. N Engl J Med 2012;367:508-19.

6 Lee EB, Fleischmann R, Hall S, et al., ORAL Start Investigators. Tofacitinib versus methotrexate in rheumatoid arthritis. $N$ Engl I Med 2014;370:2377-86.

7 Menter MA, Papp KA, Cather J, et al. Efficacy of tofacitinib for the treatment of moderate-to-severe chronic plaque psoriasis in patient subgroups from two randomised phase 3 trials. J Drugs Dermatol 2016;15:568-80.

8 Asahina A, Etoh T, Igarashi A, et al., study investigators. Oral tofacitinib efficacy, safety and tolerability in Japanese patients with moderate to severe plaque psoriasis and psoriatic arthritis: a randomized, double-blind, phase 3 study. J Dermatol 2016;43:869-80.

9 Boyle DL, Soma K, Hodge J, et al. The JAK inhibitor tofacitinib suppresses synovial JAK1-STAT signalling in rheumatoid arthritis. Ann Rheum Dis 2015;74:1311-16

10 Gao W, McGarry T, Orr C, et al. Tofacitinib regulates synovial inflammation in psoriatic arthritis, inhibiting STAT activation and induction of negative feedback inhibitors. Ann Rheum Dis 2016;75:311-15.

11 Works MG, Yin F, Yin CC, et al. Inhibition of TYK2 and JAK1 ameliorates imiquimod-induced psoriasis-like dermatitis by inhibiting IL-22 and the IL-23/IL-17 axis. J Immunol 2014;193:3278-87.

12 van der Heijde D, Dijkmans B, Geusens P, et al., Ankylosing Spondylitis Study for the Evaluation of Recombinant Infliximab Therapy Study Group. Efficacy and safety of infliximab in patients with ankylosing spondylitis: results of a randomized, placebo-controlled trial (ASSERT). Arthritis Rheum 2005;52:582-91.

13 Davis JC Jr, Van Der Heijde D, Braun J, et al., Enbrel Ankylosing Spondylitis Study Group. Recombinant human tumor necrosis factor receptor (etanercept) for treating ankylosing spondylitis: a randomized, controlled trial. Arthritis Rheum 2003;48:3230-6.

14 van der Heijde D, Kivitz A, Schiff MH, et al., ATLAS Study Group. Efficacy and safety of adalimumab in patients with ankylosing spondylitis: results of a multicenter, randomized, double-blind, placebo-controlled trial. Arthritis Rheum 2006;54:2136-46.

15 Baeten D, Sieper J, Braun J, et al., MEASURE 1 Study Group, MEASURE 2 Study Group. Secukinumab, an Interleukin-17A Inhibitor, in Ankylosing Spondylitis. N Engl J Med 2015:373:2534-48. http://dx.doi.org/10.1056/ NEJMoa1505066 